\title{
Longterm Effectiveness of Intraarticular Injections on Patient-reported Symptoms in Knee Osteoarthritis
}

\author{
Shao-Hsien Liu, Catherine E. Dubé, Charles B. Eaton, Jeffrey B. Driban, \\ Timothy E. McAlindon, and Kate L. Lapane
}

ABSTRACT. Objective. We examined the longterm effectiveness of corticosteroid or hyaluronic acid injections in relieving symptoms among persons with knee osteoarthritis (OA).

Methods. Using Osteoarthritis Initiative data, a new-user design was applied to identify participants initiating corticosteroid or hyaluronic acid injections $(n=412)$. Knee symptoms (pain, stiffness, function) were measured using The Western Ontario and McMaster Universities Osteoarthritis Index (WOMAC). We used marginal structural models adjusting for time-varying confounders to estimate the effect on symptoms of newly initiated injection use compared to nonusers over 2 years of followup. Results. Among 412 participants initiating injections, $77.2 \%$ used corticosteroid injections and 22.8\% used hyaluronic acid injections. About $18.9 \%$ had additional injection use after initiation, but switching between injection types was common. Compared to nonusers, on average, participants initiating a corticosteroid injection experienced a worsening of pain (yearly worsening: 1.24 points, $95 \%$ CI 0.82-1.66), stiffness (yearly worsening: 0.30 points, $95 \%$ CI $0.10-0.49$ ), and physical functioning (yearly worsening: 2.62 points, 95\% CI 0.94-4.29) after adjusting for potential confounders with marginal structural models. Participants initiating hyaluronic acid injections did not show improvements of WOMAC subscales (pain: $0.50,95 \%$ CI -0.11 to 1.11 ; stiffness: $-0.07,95 \%$ CI -0.38 to 0.24 ; and functioning: $0.49,95 \% \mathrm{CI}-1.34$ to 2.32 ).

Conclusion. Although intraarticular injections may support the effectiveness of reducing symptoms in short-term clinical trials, the initiation of corticosteroid or hyaluronic acid injections did not appear to provide sustained symptom relief over 2 years of followup for persons with knee OA. (First Release June 15 2018; J Rheumatol 2018;45:1316-24; doi:10.3899/jrheum.171385)

Key Indexing Terms:

INTRAARTICULAR AGENTS

KNEE OSTEOARTHRITIS

PAIN MANAGEMENT

MARGINAL STRUCTURAL MODELS

From the Clinical and Population Health Research Program, Graduate School of Biomedical Sciences, and the Division of Epidemiology of Chronic Diseases and Vulnerable Populations, Department of Quantitative Health Sciences, University of Massachusetts Medical School, Worcester, Massachusetts; Departments of Family Medicine and Epidemiology, Warren Alpert Medical School and School of Public Health, Brown University, Providence; Center for Primary Care and Prevention, Memorial Hospital of Rhode Island, Pawtucket, Rhode Island; Division of Rheumatology, Tufts Medical Center, Boston, Massachusetts, USA.

Supported by the US National Institute of Arthritis and Musculoskeletal and Skin Disease (Project number 268201000020C-1-0-1 entitled TAS::75 0888::TAS to Charles B. Eaton). The Osteoarthritis Initiative (OAI) is a public-private partnership comprising 5 contracts (N01-AR-2-2258; N01-AR-2-2259; N01-AR-2-2260; NO1-AR-2-2261; NO1-AR-2-2262) funded by the US National Institutes of Health (NIH), a branch of the US Department of Health and Human Services, and conducted by the OAI Study Investigators. Private funding partners include Merck Research Laboratories, Novartis Pharmaceuticals Corp., GlaxoSmithKline, and Pfizer Inc. Private sector funding for the OAI is managed by the Foundation for the NIH. This manuscript was prepared using an OAI public use dataset and does not necessarily reflect the opinions or views of the OAI investigators, the $\mathrm{NIH}$, or the private funding partners.

S.H. Liu, PhD, Clinical and Population Health Research Program, Graduate School of Biomedical Sciences, and the Division of Epidemiology of Chronic Diseases and Vulnerable Populations, Department of Quantitative Health Sciences, University of Massachusetts Medical School; C.E. Dubé, EdD, Division of Epidemiology of Chronic Diseases and Vulnerable Populations, Department of Quantitative Health Sciences, University of Massachusetts Medical School; C.B. Eaton, MD,
Departments of Family Medicine and Epidemiology, Warren Alpert Medical School and School of Public Health, and Center for Primary Care and Prevention, Memorial Hospital of Rhode Island; J.B. Driban, PhD, Division of Rheumatology, Tufts Medical Center; T.E. McAlindon, $M D$, Division of Rheumatology, Tufts Medical Center; K.L. Lapane, PhD, Division of Epidemiology of Chronic Diseases and Vulnerable Populations, Department of Quantitative Health Sciences, University of Massachusetts Medical School.

Address correspondence to S.H. Liu, Division of Epidemiology of Chronic Diseases and Vulnerable Populations, Department of Quantitative Health Sciences, University of Massachusetts Medical School, Worcester, Massachusetts 01605,USA.E-mail: shaohsien.liu@umassmed.edu Accepted for publication March 29, 2018.

Despite a large number of studies, the short-term safety and efficacy of intraarticular injections among persons with knee osteoarthritis (OA) remains inconclusive, leading to a lack of consensus across clinical guidelines ${ }^{1,2,3}$. Evidence about the extent to which longterm use of intraarticular injections improves patient outcomes is lacking. Systematic reviews and metaanalyses suggest that the effect of viscosupplement injections approved by the US Food and Drug Administration can last through 26 weeks, but there is no similar evidence for corticosteroid injections for persons with knee $\mathrm{OA}^{4,5,6}$. Among patients with milder disease, receiving intraarticular 
sodium hyaluronate appears to slow joint space narrowing compared to placebo ${ }^{7}$. For corticosteroid injections, there is no difference between treatment and placebo groups in joint space changes over 2 years of followup ${ }^{8}$. Outside the short-term clinical trial settings, studies documenting the longterm effect of injections on patient-reported outcomes in knee OA are scarce.

In light of the lack of evidence and conflicting practice recommendations from guidelines, it is somewhat surprising that the use of injections is increasing among Medicare (US government medical insurance for the elderly) beneficiaries newly diagnosed with knee $\mathrm{OA}^{9}$. The cost of longterm injection could be substantial (i.e., $\$ 1700$ to $\$ 3700$ for viscosupplementation treatments ${ }^{10}$. Given the widespread use of injections and the rising costs of these treatments ${ }^{10,11,12}$, understanding whether the short-term symptom relief extends to longterm effectiveness of repeated injections among persons with knee OA is warranted.

The patient population in the Osteoarthritis Initiative (OAI) provides an opportunity to research the effectiveness of injection use among persons with knee OA living in the community because this sample is more reflective of the general population with $\mathrm{OA}$ than those typically recruited in clinical trials. However, statistical inferences from observational studies are often subject to observed and/or unobserved confounding factors. In addition, when time-varying confounders (e.g., symptoms) are themselves affected by the previous treatment (e.g., injection) and thus are intermediates on the causal pathway from treatment to outcome, using the standard modeling approach may generate biased estimates of the overall treatment effect ${ }^{13,14}$. The aim of our present study was to estimate the effect of intraarticular injection use on changes in patient-reported symptoms over a 2-year period using marginal structural models to carefully adjust for time-varying confounders and intermediary variables. Through our study, we hoped to provide complementary information on patient-reported symptoms over longer periods of followup time in a more heterogeneous population compared to clinical trials so that patients and their providers would have more information about what to expect from the longterm use of these treatment options.

\section{MATERIALS AND METHODS}

The University of Massachusetts Institutional Review Board considered this study exempt because publicly available data were used.

Data source. We used publicly available data from the OAI (oai.epi-ucsf.org). The OAI was a longitudinal, multicenter, and prospective cohort study enrolling 4796 adults aged 45 to 79 years at baseline, using 4 US study sites (i.e., Baltimore, Maryland; Columbus, Ohio; Pittsburgh, Pennsylvania; and Pawtucket, Rhode Island). The aims of the OAI study were to examine the development and progression of knee OA among adults with symptomatic $\mathrm{OA}$ in at least 1 knee or at least 1 established risk factor. Participants had annual followup assessments for up to 9 years. Detailed information about the OAI protocol has been described elsewhere ${ }^{15}$.

Study sample and design. Figure 1 shows the inclusion/exclusion criteria for our study sample. Only participants with radiographically confirmed knee
$\mathrm{OA}$ in at least 1 knee at baseline [Kellgren-Lawrence (KL) grade $\geq 2$ ] were included $(n=2550)$. To improve the validity of the study, we restricted our analysis to "new users" of knee injections ${ }^{16}$. Participants who had reported injection use at baseline were not eligible $(\mathrm{n}=97)$. In addition, participants indicating no injection use but having missing values for more than half of the followup visits over the 9 years were also excluded $(n=303)$. From the remaining group, we identified participants with and without initiation of injection use during the followup period. Among initiators, we excluded those reporting use of both injection types (concurrent hyaluronic acid and corticosteroid injection users, $\mathrm{n}=52$ ), those reporting the first initiation at Year 9 because we had no followup data after the injections $(n=47)$, and those who reported injection in the affected knee after total knee replacement $(\mathrm{n}=3)$. To mimic the study design from clinical trials ${ }^{8,17}$, we further excluded participants who did not have symptomatic knee OA at baseline [Western Ontario and McMaster Universities Arthritis Index (WOMAC) pain $\geq 2$ ] as nonusers because they were considered ineligible for injection use. The final analytic sample included 412 participants initiating injection use and 576 nonusers. Among those initiating injection use with available followup information for at least 1 year, 94 initiated hyaluronic acid injections and 318 initiated corticosteroid injections.

Use of index knee. We used an index knee for the analysis based on (1) radiographic evidence of $\mathrm{OA}$, and (2) the presence of symptoms (e.g., pain) in the same knee. If only 1 knee had radiographically confirmed OA at baseline, then that knee was used as the index knee. If participants had radiographically confirmed $\mathrm{OA}$ for both knees, then the knee with higher pain scores at baseline measured by the WOMAC pain subscale was used as the index knee. If pain scores for both knees were equal, then the index knee was the one with the worse KL grade. If the aforementioned conditions were the same for the participants, we randomly selected 1 knee as the index knee.

Assessment of injection use. In OAI, injection use was assessed separately for both knees. Participants were first asked, "During the past 6 months, have you had any injections in either of your knees for treatment of arthritis?" For those answering "yes," 2 separate questions were posed regarding hyaluronic acid or corticosteroid injection use. For hyaluronic acid injections, participants were asked: "During the past 6 months, have you had an injection of hyaluronic acid (Synvisc or Hyalgan) in either of your knees for treatment of your arthritis?" These are given as a series of 3 to 5 weekly injections. To assess corticosteroid injection use, participants were asked: "During the past 6 months, have you had an injection of steroids (cortisone, corticosteroids) in either of your knees for treatment of your arthritis?" For participants whose index knees were censored during the followup (e.g., because of death, switching injection, and/or having total knee replacement), we used available information from the other knee to preserve the sample (16 out of 412).

Assessment of $O A$ symptoms. Knee symptoms were evaluated annually using the WOMAC scales (Likert version 3.1) including 3 subscales: pain $(5$ items), stiffness ( 2 items), and physical function $(17 \text { items })^{18}$. Each item of the subscale ranged from 0 to $4(0=$ none and $4=$ extreme $)$. Responses to items in each subscale were summed to produce the individual summary score ranging from $0-20$ for pain, $0-8$ for stiffness, and 0-68 for physical function. Higher WOMAC scores indicate worse symptoms/function. The primary outcome was change in each subscale between baseline visit (1 year before the injection), index visit, and 1 year after the index visit.

Covariates. We considered covariates in 2 groups: time-invariant (e.g., sociodemographic factors measured at the time of enrollment) and time-dependent [e.g., factors measured annually including clinical characteristics of OA, general health status, body mass index (BMI), and use of medications and biological supplements $]^{19}$. Multijoint symptoms were present if participants had frequent symptoms in at least 2 joints other than the knee ${ }^{20}$. Knee malalignment including varus or valgus deformity was measured and recorded using a goniometer. History of knee injuries was present if a prior injury limited the participant's ability to walk for at least 2 days, indicated on any previous visit. A history of having knee surgery was

Personal non-commercial use only. The Journal of Rheumatology Copyright @ 2018. All rights reserved. 


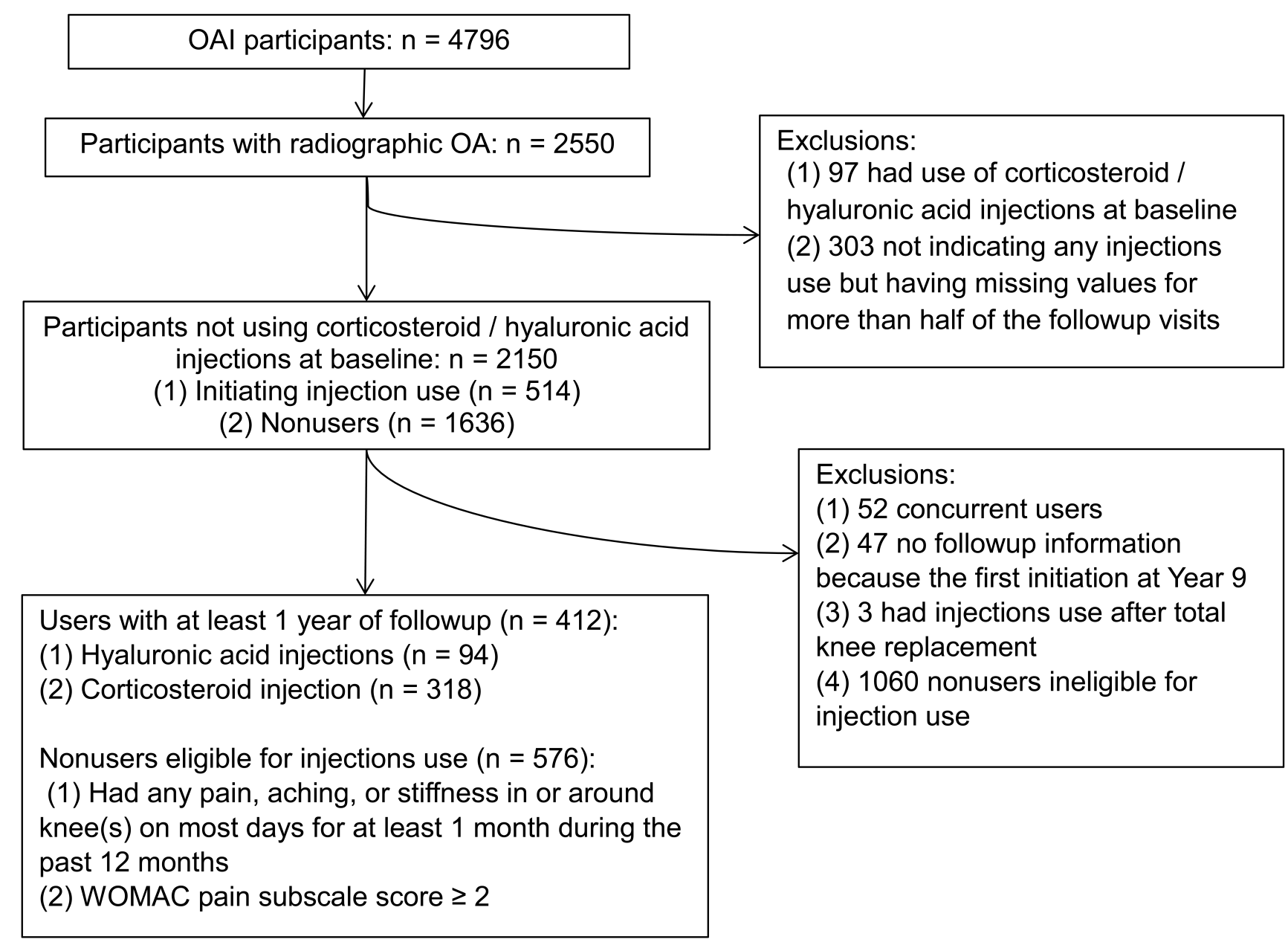

Figure 1. Flowchart of study participants. OAI: Osteoarthritis Initiative; WOMAC: Western Ontario and McMaster Universities Osteoarthritis Index.

present if participants indicated that they had arthroscopy, ligament repair, or meniscectomy on any previous visit.

The 12-item Medical Outcomes Study Short Form (SF-12) health survey was used to assess health status including physical and mental component summary scores ${ }^{21}$. Elevated depressive symptoms were considered present if participants had the Center for Epidemiologic Studies Depression Scale (CES-D) score $>16^{22}$. The Charlson Comorbidity Index was used to develop a comorbidity score and was categorized into 0,1 , and $\geq 2^{23}$. BMI was calculated from measured height and weight $\left[\right.$ weight $(\mathrm{kg}) /$ height $\left.(\mathrm{m})^{2}\right]$ and categorized as normal weight $(<25)$, overweight $(25$ to $<30)$, and obese $(\geq 30)^{24}$. Analgesic use including acetaminophen, nonsteroidal antiinflammatory drugs (NSAID), cyclooxygenase-2 selective inhibitors, opioids, and doxycycline was assessed for the previous 30 days. Biological supplements including glucosamine, chondroitin sulfate, methylsulfonylmethane, and S-adenosylmethionine were also assessed.

Statistical analysis. To understand the potential selection bias that may arise from patients lost to followup, we first compared the characteristics of sociodemographic and clinical factors and concurrent pharmacological treatment use at baseline ( $1 \mathrm{yr}$ before initiation), index year, and 1 year after initiation. We also examined the distribution of the outcome variables and ruled out departures from normality. We then developed a series of models to derive crude estimates, an estimate adjusted for baseline covariates, and adjusted for time-varying confounders using generalized estimating equations for continuous outcomes adjusted for within-participant correlation with an unstructured correlation matrix ${ }^{25}$.
Given the OAI data structure, we considered that previously measured study outcomes and time-varying confounders may be simultaneously confounders and intermediate variables (Figure 2). As a result, the estimated overall treatment effects would likely be biased using standard regression models ${ }^{26}$. To account for time-varying confounders that may lie on the causal pathway from previous treatments to the study outcomes, we used marginal structural models to estimate the overall treatment effects of injection use through inverse probability of treatment weights ${ }^{13,14}$.

The weights were calculated in 3 steps. First, we estimated time-varying stabilized inverse probability treatment weights separately for hyaluronic acid injection and corticosteroid injection use, with nonusers as the comparator at the index and followup visit. While the numerator was estimated using the conditional probability of observed injection use given the baseline characteristics, the denominator was the predicted probability of observed injection use at the index and followup visit, conditional on baseline covariates and time-varying confounders (e.g., WOMAC subscale scores measured at the prior visit and the same visit as use of injections). To construct appropriate weights, we also explored the sensitivity of weights to different model specifications at the index visit (Supplementary Table 1, available with the online version of this article $)^{27}$. For 3 different outcomes, we adjusted for the previously measured WOMAC subscales as a potential confounder. Second, because participants were censored for death, switching injection $^{28}$, and/or having total knee replacement during the followup, we estimated and incorporated the inverse probability of censoring weight to account for the potential selection bias due to differential censoring by 

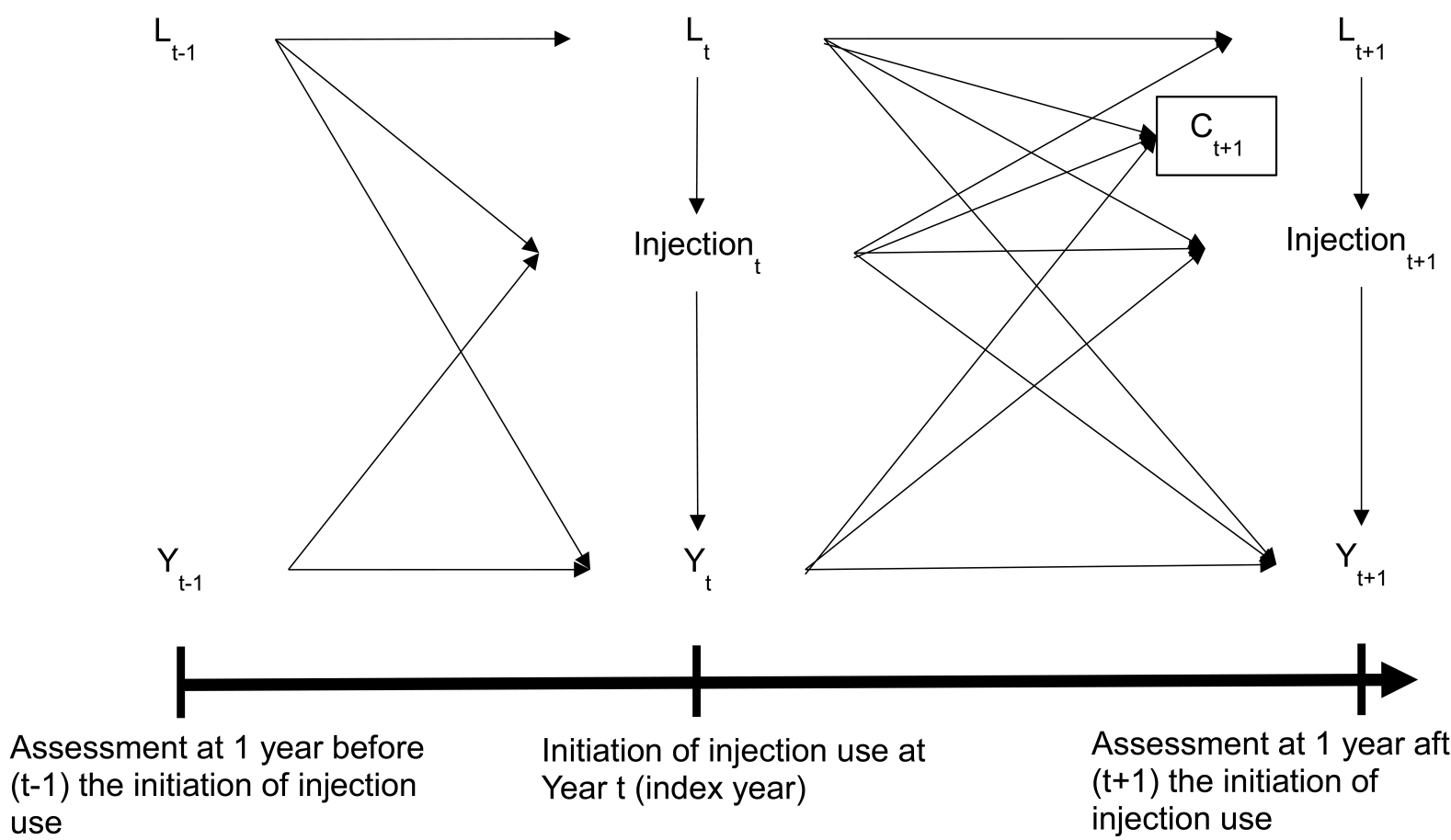

Initiation of injection use at Yeart (index year)

Figure 2. Directed acyclic graph (causal diagram) between the initiation of injection use, study outcomes, censoring, and potential time-varying confounders.

injection use ${ }^{15,14}$. Sociodemographic and clinical factors among participants who were censored by censoring mechanisms were also examined (Supplementary Table 2). Censoring weights were calculated using an approach similar to treatment weights, except that past treatment use was also added into models to estimate the probability of having observed censoring status. Lastly, final weights were then calculated as the products of treatment (including index and followup visits) and censoring weights. In addition to checking the distributions of the final weights, we also plotted the log odds of injection use conditional on the covariates to examine if there was an adequate degree of variation given observed values against the predicted injection use (Supplementary Figure 1, available with the online version of this article $)^{29}$. To minimize the effect of potential violations of the positivity assumption, we also truncated the weights at the first and 99th percentiles ${ }^{27}$.

With the final estimated weights, we used weighted linear models adjusted for baseline covariates to estimate effects of injection use on changes in symptoms with $95 \%$ CI. Under the assumptions of no unmeasured confounding with correct specifications of treatment and outcome models, the $\beta$ coefficients from marginal structural models indicated the effects of hyaluronic acid or corticosteroid injection use compared to nonusers on yearly changes in WOMAC scores. Minimal clinically important changes for improvements were defined using previous validation studies ranging from -4.6 to -1.2 for WOMAC pain, -1.5 to -0.5 for WOMAC stiffness, and -9.9 to -4.1 for WOMAC physical function $^{30,31,32,33}$.

Sensitivity analysis. To examine the robustness of our findings, we conducted sensitivity analyses to account for missing values of covariates. In particular, we were concerned about the missing data of 1 of the confounders measured at the index visit. By virtue of how we selected the sample for the current investigation and the OAI protocol, about $30 \%$ had missing data on $\mathrm{KL}$ grade. Multiple imputation was performed to handle missing data in the context of marginal structural model analyses ${ }^{34}$. We applied the Fully Conditional Specification method for imputation of missing data using SAS PROC MI FCS ${ }^{35}$. We first used all available information from the covariates (including the outcome variable) as variables in the imputation model to impute the missing values ${ }^{30}$. Twenty imputed datasets were created. We then incorporated the imputed values to rebuild the inverse probability treatment weights and fit the outcome models for each imputed dataset. Finally, we combined estimates and generated valid inferences using SAS PROC MIANALYZE to compare results.

\section{RESULTS}

Sociodemographic and clinical characteristics of study participants. Table 1 shows sociodemographic and clinical characteristics of study participants at baseline ( $1 \mathrm{yr}$ before the injection initiation), index year, and 1 year after the injection initiation among those remaining uncensored during followup. Among participants initiating injection use, the majority had $\mathrm{KL} \geq 3$. Both corticosteroid injections initiators and nonusers had similar distribution of sociodemographic and clinical characteristics at baseline. Men and those with higher household income (e.g., $>\$ 50,000$ ) compose the majority of hyaluronic acid injection initiators relative to nonusers. Among hyaluronic acid injection initiators, 33.7\% had KL grade 4, while $17.4 \%$ of nonusers had KL grade 4 . During followup, the distribution of characteristics was similar over time compared to the distribution at baseline. The proportion of those censored at 1 year after initiation was $29.8 \%$ for hyaluronic acid injection initiators relative to the other groups (e.g., corticosteroid 19.9\%; nonusers 3.0\%).

Concurrent pharmacological treatment use. NSAID were the most commonly reported concurrent-use pharmacological treatments among the study groups at baseline (Table 2). The majority of injection initiators reported analgesic use. Similar to sociodemographic and clinical characteristics, both corti-

Personal non-commercial use only. The Journal of Rheumatology Copyright (C) 2018. All rights reserved. 
Table 1. Sociodemographic and clinical characteristics among participants with radiographically confirmed knee OA by use of injections. Values are $\%$ or mean \pm SD unless otherwise specified.

\begin{tabular}{|c|c|c|c|c|c|c|c|c|c|}
\hline \multirow[t]{2}{*}{ Characteristics } & \multicolumn{3}{|c|}{ Baseline $^{\mathrm{a}}$} & \multicolumn{3}{|c|}{ Index Year } & \multicolumn{3}{|c|}{ One Year after Injection Initiation } \\
\hline & $\mathrm{CO}$ & HA & Nonuser & $\mathrm{CO}$ & HA & Nonuser & $\mathrm{CO}$ & HA & Nonuser \\
\hline Total, $\mathrm{n}^{*}$ & 318 & 94 & 576 & 318 & 94 & 576 & 257 & 66 & 559 \\
\hline \multicolumn{10}{|l|}{ Proportions relative } \\
\hline to baseline & 100 & 100 & 100 & 100 & 100 & 100 & 80.1 & 70.2 & 97.0 \\
\hline Injection use, $\mathrm{n}$ & 0 & 0 & 0 & 318 & 94 & 0 & 63 & 15 & 0 \\
\hline Age, yrs & $66.9 \pm 9.2$ & $65.0 \pm 8.6$ & $64.0 \pm 9.3$ & $67.9 \pm 9.2$ & $66.0 \pm 8.6$ & $65.0 \pm 9.3$ & $68.5 \pm 9.1$ & $66.0 \pm 8.8$ & $65.8 \pm 9.4$ \\
\hline Women & 64.2 & 44.7 & 55.6 & 64.2 & 44.7 & 55.6 & 65.4 & 43.9 & 55.5 \\
\hline \multicolumn{10}{|l|}{ Ethnicity/race } \\
\hline Non-Hispanic white & 77.4 & 88.3 & 67.9 & 77.4 & 88.3 & 67.9 & 75.5 & 87.9 & 67.4 \\
\hline Non-Hispanic black & 17.9 & 6.4 & 30.0 & 17.9 & 6.4 & 30.0 & 21.0 & 7.6 & 30.4 \\
\hline Other & 4.7 & 5.3 & 2.1 & 4.7 & 5.3 & 2.1 & 3.5 & 4.6 & 2.2 \\
\hline \multicolumn{10}{|l|}{ Education } \\
\hline High school or less & 18.6 & 12.9 & 20.9 & 18.6 & 12.9 & 20.9 & 20.2 & 10.8 & 20.2 \\
\hline Some college & 27.1 & 23.7 & 26.7 & 27.1 & 23.7 & 26.7 & 28.8 & 24.6 & 27.1 \\
\hline College graduate & 22.1 & 20.4 & 19.2 & 22.1 & 20.4 & 19.2 & 19.5 & 18.5 & 19.8 \\
\hline Graduate school & 32.2 & 43.0 & 33.3 & 32.2 & 43.0 & 33.3 & 31.5 & 46.2 & 32.9 \\
\hline \multicolumn{10}{|l|}{ Income (US\$) } \\
\hline$<25,000$ & 15.5 & 4.3 & 17.9 & 15.5 & 4.3 & 17.9 & 17.1 & 4.6 & 18.1 \\
\hline $25,000-50,000$ & 30.9 & 22.6 & 27.8 & 30.9 & 22.6 & 27.8 & 32.7 & 16.9 & 27.6 \\
\hline$>50,000$ & 53.6 & 73.1 & 54.3 & 53.6 & 73.1 & 54.3 & 50.2 & 78.5 & 54.3 \\
\hline \multicolumn{10}{|l|}{ KL grade } \\
\hline 2 & 40.1 & 22.1 & 52.3 & 35.7 & 16.3 & 50.0 & 38.9 & 22.2 & 50.7 \\
\hline 3 & 39.1 & 44.2 & 30.3 & 37.9 & 45.0 & 30.8 & 41.2 & 46.3 & 31.2 \\
\hline 4 & 20.8 & 33.7 & 17.4 & 26.4 & 38.8 & 19.3 & 19.9 & 31.5 & 18.2 \\
\hline \multicolumn{10}{|l|}{ Symptom-related } \\
\hline multijoint $\mathrm{OA}$ & 55.4 & 57.5 & 55.2 & 55.7 & 60.6 & 50.4 & 56.0 & 62.1 & 49.0 \\
\hline History of knee injury & 40.6 & 57.5 & 49.3 & 47.2 & 58.5 & 50.2 & 49.4 & 57.6 & 51.2 \\
\hline History of knee surgery & 28.6 & 48.9 & 34.6 & 32.1 & 54.3 & 35.8 & 35.8 & 50.0 & 35.2 \\
\hline \multicolumn{10}{|l|}{$\mathrm{BMI}, \mathrm{kg} / \mathrm{m}^{2}$} \\
\hline$<25$ & 12.9 & 14.9 & 13.2 & 12.9 & 16.0 & 13.0 & 12.1 & 15.2 & 14.5 \\
\hline 25 to $<30$ & 40.6 & 31.9 & 39.7 & 40.6 & 30.9 & 37.7 & 40.5 & 31.8 & 36.3 \\
\hline$\geq 30$ & 46.5 & 53.2 & 47.1 & 46.5 & 53.2 & 49.3 & 47.5 & 53.0 & 49.2 \\
\hline \multicolumn{10}{|l|}{ Knee alignment } \\
\hline Normal & 20.5 & 18.9 & 18.4 & 17.4 & 15.9 & 15.9 & 18.4 & 11.9 & 16.0 \\
\hline Varus & 39.3 & 40.0 & 41.9 & 42.9 & 44.3 & 45.9 & 38.2 & 44.1 & 46.5 \\
\hline Valgus & 40.3 & 41.1 & 39.7 & 39.7 & 39.8 & 38.2 & 43.4 & 44.1 & 37.5 \\
\hline CES-D (> 16) & 11.0 & 8.9 & 11.0 & 14.3 & 12.2 & 13.3 & 13.2 & 12.9 & 12.1 \\
\hline \multicolumn{10}{|l|}{ Charlson CI } \\
\hline 0 & 65.4 & 70.2 & 65.7 & 62.0 & 71.0 & 63.4 & 56.7 & 78.5 & 63.0 \\
\hline 1 & 18.2 & 22.3 & 19.3 & 19.9 & 20.4 & 19.9 & 21.7 & 15.4 & 19.9 \\
\hline$\geq 2$ & 16.4 & 7.5 & 15.1 & 18.0 & 8.6 & 16.7 & 21.7 & 6.2 & 17.1 \\
\hline WOMAC pain & $5.0 \pm 3.9$ & $5.1 \pm 3.9$ & $4.9 \pm 4.1$ & $6.2 \pm 4.2$ & $5.7 \pm 3.6$ & $4.4 \pm 3.9$ & $5.6 \pm 4.0$ & $5.2 \pm 3.7$ & $4.2 \pm 3.8$ \\
\hline WOMAC stiffness & $2.7 \pm 1.7$ & $2.5 \pm 1.7$ & $2.5 \pm 1.8$ & $2.8 \pm 1.7$ & $2.8 \pm 1.6$ & $2.4 \pm 1.8$ & $2.7 \pm 1.7$ & $2.5 \pm 1.6$ & $2.2 \pm 1.8$ \\
\hline \multicolumn{10}{|l|}{ WOMAC physical } \\
\hline function & $16.8 \pm 13.0$ & $16.7 \pm 11.8$ & $15.3 \pm 13.0$ & $19.2 \pm 13.2$ & $17.1 \pm 11.3$ & $14.4 \pm 12.8$ & $17.9 \pm 13.2$ & $16.9 \pm 11.1$ & $14.1 \pm 12.8$ \\
\hline KOOS-QoL & $54.6 \pm 19.9$ & $52.2 \pm 18.8$ & $56.0 \pm 21.7$ & $48.3 \pm 20.2$ & $49.0 \pm 20.1$ & $57.4 \pm 22.4$ & $50.6 \pm 20.3$ & $50.5 \pm 19.5$ & $58.4 \pm 22.0$ \\
\hline SF-12 physical & & & & & & & & & \\
\hline component score & $42.8 \pm 9.6$ & $42.7 \pm 9.2$ & $44.8 \pm 9.7$ & $40.8 \pm 9.4$ & $40.8 \pm 9.9$ & $44.4 \pm 10.1$ & $41.1 \pm 9.5$ & $40.6 \pm 9.3$ & $44.4 \pm 10.0$ \\
\hline SF-12 mental & & & & & & & & & \\
\hline component score & $54.1 \pm 8.4$ & $55.4 \pm 7.1$ & $53.8 \pm 8.3$ & $54.1 \pm 9.5$ & $55.4 \pm 8.2$ & $53.4 \pm 9.3$ & $53.6 \pm 9.1$ & $55.1 \pm 8.5$ & $53.2 \pm 9.2$ \\
\hline Joint space width, mm & $4.6 \pm 1.9$ & $4.2 \pm 2.2$ & $5.1 \pm 1.6$ & $4.4 \pm 1.9$ & $4.0 \pm 2.1$ & $4.9 \pm 1.7$ & $4.4 \pm 1.9$ & $4.3 \pm 2.1$ & $4.9 \pm 1.6$ \\
\hline
\end{tabular}

a One year before the index year. * No. participants with missing information: At baseline - education (4), income (3), KL grade (114), body mass index (1), knee alignment (99), CES-D (63), Charlson Comorbidity Index (5), WOMAC pain (29), WOMAC stiffness (27), WOMAC physical function (3), KOOS-QoL (27), SF-12 physical component score (70), SF-12 mental component score (70), joint space width (234). At index year - education (4), income (3), KL grade (153), knee alignment (123), CES-D (72), Charlson Comorbidity Index (5), WOMAC pain (25), WOMAC stiffness (27), WOMAC physical function (41), KOOS-QoL (27), SF-12 physical component score (93), SF-12 mental component score (93), joint space width (300). One year after injection initiation education (3), income (2), KL grade (145), knee alignment (120), CES-D (80), Charlson Comorbidity Index (6), WOMAC Pain (56), WOMAC Stiffness (56), WOMAC Physical function (71), KOOS-QoL (56), SF-12 Physical Component Score (93), SF-12 Mental Component Score (93), joint space width (292). OA: osteoarthritis; CO: corticosteroid; HA: hyaluronic acid; KL grade: the Kellgren-Lawrence grade; CES-D: Center for Epidemiologic Studies Depression Scale; WOMAC: The Western Ontario and McMaster Universities Osteoarthritis Index; KOOS-QoL: Knee Injury and Osteoarthritis Outcome Score quality of life subscale; SF-12: 12-item Medical Outcomes Study Short Form; CI: Comorbidity Index; BMI: body mass index. 
Table 2. Concomitant use of medications and supplements among persons with radiographically confirmed knee OA by the use of injections.

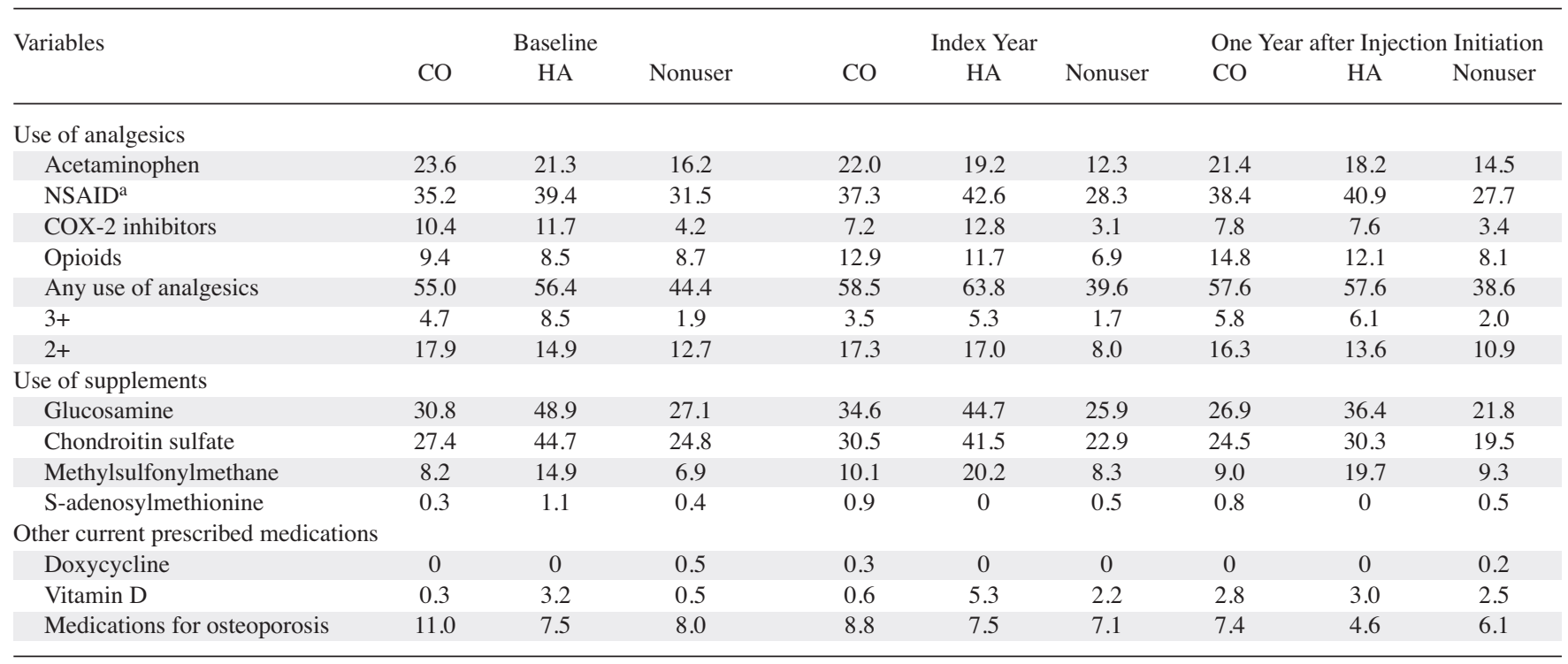

Values are percentages. ${ }^{\text {a }}$ Including self-reported over-the-counter use and current prescriptions such as aspirin, ibuprofen, and salicylate. No. participants with missing information: At baseline - NSAID (29), doxycycline (27), Vitamin D (27). At index year - NSAID (31), doxycycline (26), Vitamin D (31). One year after injection initiation - NSAID (58), doxycycline (40), Vitamin D (43). NSAID: nonsteroidal antiinflammatory drugs; CO: corticosteroid; HA: hyaluronic acid; OA: osteoarthritis; COX: cyclooxygenase.

costeroid injection initiators and nonusers had similar distributions of concurrent pharmacological treatment use at baseline. Among hyaluronic acid injection initiators, $48.9 \%$ reported concurrent use of supplements such as glucosamine, whereas corticosteroid injection initiators and nonusers reported $30.8 \%$ and $27.1 \%$, respectively. During followup, the distribution of concurrent pharmacological treatments use remained similar over time between corticosteroid injection initiators and the nonusers group. However, among hyaluronic acid injection initiators, the use of glucosamine or chondroitin sulfate decreased from baseline to 1 year after initiation (e.g., glucosamine $48.9 \%$ to $36.4 \%$; chondroitin sulfate $44.7 \%$ to $30.3 \%$ ).

Effects of injection use on knee OA. Table 3 shows average effects of initiating corticosteroid or hyaluronic acid injections compared to nonusers on patient-reported outcomes. After adjusting for potential confounders with marginal structural models, the use of corticosteroid injections did not improve WOMAC subscales compared with nonusers. On average, the yearly changes were 1.24 (95\% CI 0.82-1.66) for WOMAC pain, 0.30 (95\% CI 0.10-0.49) for WOMAC stiffness, and 2.62 (95\% CI 0.94-4.29) for WOMAC physical function. While results from sensitivity analyses were qualitatively similar to our main findings, the effect of estimates for WOMAC pain did not meet a priori definitions of minimal clinically important differences.

While the use of hyaluronic acid injections did not show improvements of WOMAC subscales compared with nonusers, the magnitude of effects was relatively smaller compared to corticosteroid injection use. On average, the yearly changes were 0.50 (95\% CI -0.11 to 1.11 ) for WOMAC pain, -0.07 (95\% CI -0.38 to 0.24 ) for WOMAC stiffness, and 0.49 (95\% CI -1.34 to 2.32 ) for WOMAC physical function. The findings from sensitivity analyses remained similar.

\section{DISCUSSION}

Using data from the OAI, we did not observe reduced symptoms associated with the initiation of corticosteroid or hyaluronic acid injections compared with nonusers among participants with radiographically confirmed knee $\mathrm{OA}$ in the 2 years of followup after carefully controlling for potential time-varying and time-independent confounders with marginal structural models.

Among participants initiating corticosteroid injection use compared to nonusers, our study findings are consistent with an updated review ${ }^{4}$. However, the study duration included in the review varied (range: 2 wks- $-1 \mathrm{yr}$ ) and mixed with single and/or multiple injection use. To our knowledge, there are only 2 published trials that are comparable to our study design, which assessed the effect of continuous intraarticular corticosteroid use over 2 years. Our findings are consistent with both studies and demonstrate that the use of corticosteroid injections does not appear to reduce symptoms ${ }^{8,37}$. Although more studies with adequate power and proper design may still be needed, our findings do contribute to the growing body of evidence produced using nonexperimental study design with advanced analytical techniques.

Our results did not appear to support the notion that initiating hyaluronic acid injection use would be effective at

Personal non-commercial use only. The Journal of Rheumatology Copyright $\subset$ 2018. All rights reserved. 
Table 3. Estimated effects on symptoms of injection use compared with nonusers among persons with radiographically confirmed knee OA.

\begin{tabular}{|c|c|c|}
\hline WOMAC Subscale & $\begin{array}{c}\text { Use of } \mathrm{CO}, \beta^{*} \\
\text { coefficient }(95 \% \mathrm{CI})\end{array}$ & $\begin{array}{c}\text { Use of HA, } \beta^{*} \\
\text { coefficient }(95 \% \mathrm{CI})\end{array}$ \\
\hline \multicolumn{3}{|l|}{ Pain } \\
\hline Crude ${ }^{\mathrm{a}}$ & $1.17(0.80$ to 1.54$)$ & $0.77(0.17$ to 1.38$)$ \\
\hline Baseline covariates $^{\mathrm{b}}$ & $3.49(-2.36$ to 9.33$)$ & $0.58(0.07$ to 1.09$)$ \\
\hline Baseline plus time-varying covariates ${ }^{\mathrm{c}}$ & $2.11(0.70$ to 3.53$)$ & $0.40(-0.30$ to 1.10$)$ \\
\hline Marginal structural model ${ }^{\mathrm{d}}$ & $1.24(0.82$ to 1.66$)$ & $0.50(-0.11$ to 1.11$)$ \\
\hline Sensitivity analysis & $0.51(-0.31$ to 1.32$)$ & $0.42(-1.44$ to 2.29$)$ \\
\hline \multicolumn{3}{|l|}{ Stiffness } \\
\hline Crude $^{\mathrm{a}}$ & 0.23 (0.04 to 0.43$)$ & $0.28(-0.03$ to 0.59$)$ \\
\hline Baseline covariates $^{\mathrm{b}}$ & $-0.55(-1.27$ to 0.17$)$ & $-2.10(-2.35$ to -1.85$)$ \\
\hline Baseline plus time-varying covariates ${ }^{\mathrm{c}}$ & $0.05(-1.08$ to 1.18$)$ & $0.17(-0.17$ to 0.51$)$ \\
\hline Marginal structural model ${ }^{\mathrm{d}}$ & $0.30(0.10$ to 0.49$)$ & $-0.07(-0.38$ to 0.24$)$ \\
\hline Sensitivity analysis & $0.14(-0.21$ to 0.50$)$ & $-0.43(-1.54$ to 0.67$)$ \\
\hline \multicolumn{3}{|l|}{ Physical function } \\
\hline Crude ${ }^{a}$ & $2.60(1.36$ to 3.84$)$ & $1.21(-0.70$ to 3.12$)$ \\
\hline Baseline covariates $^{\mathrm{b}}$ & $-1.73(-9.50$ to 6.05$)$ & $1.16(-0.50$ to 2.83$)$ \\
\hline Baseline plus time-varying covariates ${ }^{\mathrm{c}}$ & $0.06(-0.51$ to 0.62$)$ & $-0.37(-2.45$ to 1.71$)$ \\
\hline Marginal structural model ${ }^{\mathrm{d}}$ & $2.62(0.94$ to 4.29$)$ & $0.49(-1.34$ to 2.32$)$ \\
\hline Sensitivity analysis & $1.05(-1.23$ to 3.33$)$ & $3.84(-3.72$ to 11.40$)$ \\
\hline
\end{tabular}

\footnotetext{
* A negative $\beta$ coefficient indicates improvement for the WOMAC subscales and worsening for the joint space

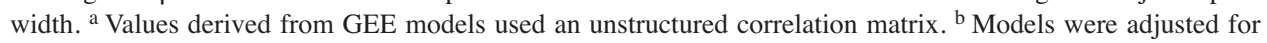
baseline characteristics including age, sex, race/ethnicity, education, income, KL grade, body mass index, history of knee injury, history of knee surgery, SF-12 physical and mental component summary scores, WOMAC subscales, and use of analgesics and glucosamine. ${ }^{\mathrm{c}}$ In addition to baseline covariates, time-varying confounders including KL grade, history of knee injury and surgery, WOMAC subscales, and SF-12 physical and mental component summary scores measured at the same visit as injection use were also adjusted. ${ }^{\mathrm{d}}$ Inverse probability-weighted analyses with truncated weights were used. OA: osteoarthritis; CO: corticosteroid; GEE: generalized estimating equations; HA: hyaluronic acid; WOMAC: Western Ontario and McMaster Universities Arthritis Index; KL: Kellgren-Lawrence; SF-12: 12-item Medical Outcomes Study Short Form.
}

relieving symptoms over longer periods of time ( $2 \mathrm{yrs})$. Although our findings are not consistent with evidence from some reviews and a metaanalysis ${ }^{5,38}$, there are some issues that may hamper the comparison. First, the followup periods in trials included in the reviews are mostly short-term, with only 1 treatment cycle. Thus, the beneficial effects of longterm use remain unclear. In addition, the potential efficacy of hyaluronic acid injections to patients with more severe disease remains unknown because some trials excluded patients with severe knee OA. In our study, we observed that a substantial percentage of participants initiating these injections had KL 4. It may well be that hyaluronic acid injections are more effective during earlier stages of disease. Further, when comparing results from larger trials with better quality, later updated reviews suggested that the use of hyaluronic acid injections compared to nonusers is associated with small but not clinically important improvement in knee symptoms ${ }^{39,40}$. Indeed, for changes in symptoms, our results are consistent with a study with a larger sample size over 1 year of followup ${ }^{41}$.

Our study builds on previous research in several areas. First, we used data from the OAI, a cohort study that enrolled participants with knee OA living in the community and conducted annual assessments with validated patient-reported outcomes and measures of disease progression. We were thus able to evaluate the effectiveness of injection use in the real-world setting. With detailed information regarding disease severity, we included comparable participants who did not receive injections to reduce confounding by indication. Second, compared to clinical trials ${ }^{4,5}$, this longitudinal and nonexperimental study enabled us to examine injection use over a longer period and to evaluate treatment benefits in a real-world setting. While the typical clinical trial in patients with OA lasts about 6 months, we followed patients for 2 years. Lastly, to address threats to the validity of our study such as time-varying confounders and loss to followup, we used advanced statistical techniques of inverse probability treatment/censoring weights with marginal structural models ${ }^{14}$. We further performed sensitivity analyses using multiple imputation to evaluate the robustness of the main results. Results from sensitivity analyses showed consistent findings.

Several limitations are also acknowledged. No information was available on the formulation of injections as well as dosages used ${ }^{40}$. Given the questionnaires used in the OAI, there is a potential for mismatch between the time of injection use and outcome assessments. For example, at annual assessment visits when participants were asked about injec-

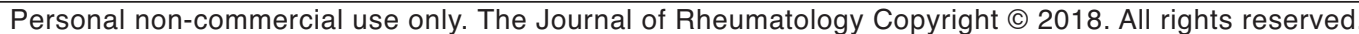


tions used, they could be in the middle of a treatment cycle. On the other hand, misclassification of participants as nonusers is possible. In addition, whether the use of injection was due to pain fluctuation or flares 42,43 and thus whether patients may experience clinically meaningful improvement remains unknown. Confounding by indication is still a possibility despite the comprehensive assessments of disease severity and concurrent treatment use in the OAI. Although sensitivity analyses were conducted due to the concern of missing data on some key covariates (e.g., KL grade), little guidance exists regarding how to best handle missing data in the context of marginal structural models ${ }^{34,44}$. Lastly, in the practice of constructing appropriate weights, there could be a model misspecification and/or violation of positivity assumption $^{27}$. However, we carefully constructed weights using an iterative process and graphically examined whether there was an adequate degree of variation given observed and predicted injection use. We also truncated weights to reduce the potential effect of violating the positivity assumption ${ }^{27}$.

Initiating treatments with either corticosteroid or hyaluronic acid injections was not associated with reduced symptoms compared to nonusers over 2 years in patients with knee OA. In addition to the substantial cost from longterm use $^{10}$, patients initiating such treatment options should be advised that the beneficial effects of such treatments may be time-limited. Future research targeting comparative effectiveness of these commonly used injections may be helpful to understand the effectiveness of these treatments for patients with knee OA.

\section{ONLINE SUPPLEMENT}

Supplementary material accompanies the online version of this article.

\section{REFERENCES}

1. Hochberg MC, Altman RD, April KT, Benkhalti M, Guyatt G, McGowan J, et al. American College of Rheumatology 2012 recommendations for the use of nonpharmacologic and pharmacologic therapies in osteoarthritis of the hand, hip, and knee. Arthritis Care Res 2012;64:465-74.

2. Jevsevar DS. Treatment of osteoarthritis of the knee: evidence-based guideline, 2nd edition. J Am Acad Orthop Surg 2013;21:571-6.

3. McAlindon TE, Bannuru RR, Sullivan MC, Arden NK, Berenbaum F, Bierma-Zeinstra SM, et al. OARSI guidelines for the non-surgical management of knee osteoarthritis. Osteoarthritis Cartilage 2014;22:363-88.

4. Jüni P, Hari R, Rutjes AW, Fischer R, Silletta MG, Reichenbach S, et al. Intra-articular corticosteroid for knee osteoarthritis. Cochrane Database Syst Rev 2015;CD005328.

5. Strand V, McIntyre LF, Beach WR, Miller LE, Block JE. Safety and efficacy of US-approved viscosupplements for knee osteoarthritis: a systematic review and meta-analysis of randomized, saline-controlled trials. J Pain Res 2015;8:217-28.

6. Bannuru RR, Natov NS, Obadan IE, Price LL, Schmid CH, McAlindon TE. Therapeutic trajectory of hyaluronic acid versus corticosteroids in the treatment of knee osteoarthritis: a systematic review and meta-analysis. Arthritis Rheum 2009;61:1704-11.

7. Jubb RW, Piva S, Beinat L, Dacre J, Gishen P. A one-year, randomised, placebo (saline) controlled clinical trial of 500-730
$\mathrm{kDa}$ sodium hyaluronate (Hyalgan) on the radiological change in osteoarthritis of the knee. J Clin Pract 2003;57:467-74.

8. Raynauld JP, Buckland-Wright C, Ward R, Choquette D, Haraoui B, Martel-Pelletier J, et al. Safety and efficacy of long-term intraarticular steroid injections in osteoarthritis of the knee: a randomized, double-blind, placebo-controlled trial. Arthritis Rheum 2003;48:370-7.

9. Koenig KM, Ong KL, Lau EC, Vail TP, Berry DJ, Rubash HE, et al. The use of hyaluronic acid and corticosteroid injections among Medicare patients with knee osteoarthritis. J Arthroplasty 2016;31:351-5.

10. Arnold W, Fullerton DSP, Holder S, May CS. Viscosupplementation: managed care issues for osteoarthritis of the knee. J Manag Care Pharm 2007;13 (4 Suppl):S3-19.

11. Wen DY. Intra-articular hyaluronic acid injections for knee osteoarthritis. Am Fam Physician 2000;62:565-70.

12. Watterson JR, Esdaile JM. Viscosupplementation: therapeutic mechanisms and clinical potential in osteoarthritis of the knee. J Am Acad Orthop Surg 2000;8:277-84.

13. Hernán MA, Brumback B, Robins JM. Marginal structural models to estimate the causal effect of zidovudine on the survival of HIV-positive men. Epidemiology 2000;11:561-70.

14. Robins JM, Hernán MA, Brumback B. Marginal structural models and causal inference in epidemiology. Epidemiology 2000; 11:550-60.

15. Nevitt MC, Felson DT, Lester G. The Osteoarthritis Initiative protocol for the cohort study. 2006. [Internet. Accessed May 24, 2018.] Available from: oai.epiucsf.org/datarelease/docs/StudyDesignProtocol.pdf

16. Ray WA. Evaluating medication effects outside of clinical trials: new-user designs. Am J Epidemiol 2003;158:915-20.

17. Leopold SS, Redd BB, Warme WJ, Wehrle PA, Pettis PD, Shott S Corticosteroid compared with hyaluronic acid injections for the treatment of osteoarthritis of the knee. A prospective, randomized trial. J Bone Joint Surg Am 2003;85-A:1197-203.

18. Roos EM, Klässbo M, Lohmander LS. WOMAC osteoarthritis index. Reliability, validity, and responsiveness in patients with arthroscopically assessed osteoarthritis. Western Ontario and MacMaster Universities. Scand J Rheumatol 1999;28:210-5.

19. Lapane KL, Liu SH, Dubé CE, Driban JB, McAlindon TE, Eaton $\mathrm{CB}$. Factors associated with the use of hyaluronic acid and corticosteroid injections among patients with radiographically confirmed knee osteoarthritis: a retrospective data analysis. Clin Ther 2017;39:347-58.

20. Okma-Keulen P, Hopman-Rock M. The onset of generalized osteoarthritis in older women: a qualitative approach. Arthritis Rheum 2001;45:183-90.

21. Ware J, Kosinski M, Keller SD. A 12-item short-form health survey: construction of scales and preliminary tests of reliability and validity. Med Care 1996;34:220-33.

22. Radloff LS. The CES-D Scale: A self-report depression scale for research in the general population. Appl Psychol Meas 1977; 1:385-401.

23. Katz JN, Chang LC, Sangha O, Fossel AH, Bates DW. Can comorbidity be measured by questionnaire rather than medical record review? Med Care 1996;34:73-84.

24. Jensen MD, Ryan DH, Apovian CM, Ard JD, Comuzzie AG, Donato KA, et al. 2013 AHA/ACC/TOS guideline for the management of overweight and obesity in adults: a report of the American College of Cardiology/American Heart Association Task Force on Practice Guidelines and The Obesity Society. Circulation 2014;129:S102-38.

25. Platt RW, Brookhart MA, Cole SR, Westreich D, Schisterman EF. An information criterion for marginal structural models. Stat Med 2013;32:1383-93.

Personal non-commercial use only. The Journal of Rheumatology Copyright @ 2018 . All rights reserved. 
26. Hernán MA, Hernández-Díaz S, Robins JM. A structural approach to selection bias. Epidemiology 2004;15:615-25.

27. Cole SR, Hernán MA. Constructing inverse probability weights for marginal structural models. Am J Epidemiol 2008;168:656-64.

28. Liu SH, Dubé CE, Driban JB, McAlindon TE, Eaton CB, Lapane KL. Patterns of intra-articular injection use after initiation of treatment in patients with knee osteoarthritis: data from the osteoarthritis initiative. Osteoarthritis Cartilage 2017;25:1607-14.

29. Mortimer KM, Neugebauer R, Van Der Laan M, Tager IB. An application of model-fitting procedures for marginal structural models. Am J Epidemiol 2005;162:382-8.

30. Vignon E, Piperno M, Le Graverand MP, Mazzuca SA, Brandt KD, Mathieu P, et al. Measurement of radiographic joint space width in the tibiofemoral compartment of the osteoarthritic knee: comparison of standing anteroposterior and Lyon schuss views. Arthritis Rheum 2003;48:378-84.

31. Dougados M, Hawker G, Lohmander S, Davis AM, Dieppe P, Maillefert JF, et al. OARSI/OMERACT criteria of being considered a candidate for total joint replacement in knee/hip osteoarthritis as an endpoint in clinical trials evaluating potential disease modifying osteoarthritic drugs. J Rheumatol 2009;36:2097-9.

32. Angst F, Aeschlimann A, Stucki G. Smallest detectable and minimal clinically important differences of rehabilitation intervention with their implications for required sample sizes using WOMAC and SF-36 quality of life measurement instruments in patients with osteoarthritis of the lower extremities. Arthritis Rheum 2001;45:384-91.

33. Greco NJ, Anderson AF, Mann BJ, Cole BJ, Farr J, Nissen CW, et al. Responsiveness of the International Knee Documentation Committee Subjective Knee Form in comparison to the Western Ontario and McMaster Universities Osteoarthritis Index, modified Cincinnati Knee Rating System, and Short Form 36 in patients with focal articular cartilage defects. Am J Sports Med 2010;38:891-902.

34. Moodie EE, Delaney JA, Lefebvre G, Platt RW. Missing confounding data in marginal structural models: a comparison of inverse probability weighting and multiple imputation. Int J Biostat 2008;4:1-23.
35. White IR, Royston P, Wood AM. Multiple imputation using chained equations: Issues and guidance for practice. Stat Med 2011; 30:377-99.

36. Moons KG, Donders RA, Stijnen T, Harrell FE Jr. Using the outcome for imputation of missing predictor values was preferred. J Clin Epidemiol 2006;59:1092-101.

37. McAlindon TE, LaValley MP, Harvey WF, Price LL, Driban JB, Zhang $\mathrm{M}$, et al. Effect of intra-articular triamcinolone vs saline on knee cartilage volume and pain in patients with knee osteoarthritis: A randomized clinical trial. JAMA 2017;317:1967-75.

38. Bellamy N, Campbell J, Robinson V, Gee T, Bourne R, Wells G. Viscosupplementation for the treatment of osteoarthritis of the knee. Cochrane database Syst Rev 2006;CD005321.

39. Rutjes AW, Juni P, da Costa BR, Trelle S, Nuesch E, Reichenbach S Viscosupplementation for osteoarthritis of the knee: a systematic review and meta-analysis. Ann Intern Med 2012;157:180-91.

40. Jevsevar D, Donnelly P, Brown GA, Cummins DS . Viscosupplementation for osteoarthritis of the knee. J Bone Joint Surg Am 2015;97:2047-60.

41. Pham T, Le Henanff A, Ravaud P, Dieppe P, Paolozzi L, Dougados M. Evaluation of the symptomatic and structural efficacy of a new hyaluronic acid compound, NRD101, in comparison with diacerein and placebo in a 1 year randomised controlled study in symptomatic knee osteoarthritis. Ann Rheum Dis 2004;63:1611-7.

42. Neogi T, Nevitt MC, Yang M, Curtis JR, Torner J, Felson DT. Consistency of knee pain: correlates and association with function. Osteoarthritis Cartilage 2010;18:1250-5.

43. Parry E, Ogollah R, Peat G. Significant pain variability in persons with, or at high risk of, knee osteoarthritis: preliminary investigation based on secondary analysis of cohort data. BMC Musculoskelet Disord 2017;18:1-11.

44. Vourli G, Touloumi G. Performance of the marginal structural models under various scenarios of incomplete marker's values: a simulation study. Biometrical J 2015;57:254-70. 